\title{
DIABETES
}

\section{Drug-eluting balloons for the treatment of critical limb ischaemia}

Patients with diabetes mellitus are at risk of peripheral artery disease as a result of atherosclerotic disease in blood vessels below the knee. Critical limb ischaemia is an advanced form of peripheral artery disease. In the DEBATE-BTK trial, 1 year rates of restenosis were compared in patients with diabetes and critical limb ischaemia who were treated with either drug-eluting balloons (DEB) or conventional percutaneous transluminal angioplasty (PTA). Restenosis occurred substantially less frequently in patients treated with DEB than in those treated with PTA ( $27 \%$ and $74 \%$ respectively, $P<0.001$ ).

Critical limb ischaemia is associated with high morbidity and mortality. The DEBATE-BTK investigators had "observed that the rate of target lesion revascularization in patients treated by conventional balloon angioplasty in below-the-knee lesions remained unacceptable (around 30-50\%)," despite strict clinical surveilance of these patients, says Francesco Liistro, lead author of the DEBATE-BTK trial report. "We elected to focus our trial on the preservation of vessel patency, as repeat interventions with multiple contrast exposures are harmful to these very sick patients, [many of whom have] life-threatening comorbidities," he says. A total of 132 patients with long stenoses $(\geq 40 \mathrm{~mm})$ in at least one tibial vessel were randomly assigned to receive treatment with either DEB (65 patients with 80 lesions in 71 limbs) or PTA (67 patients with 78 lesions in 72 limbs). Restenosis was determined by angiography 12 months after the procedure.

The rates of both target lesion revascularization and target lesion occlusion were significantly lower in patients treated with DEB than in those treated with PTA (18\% and $43 \%$ respectively for target lesion revascularization, $P=0.002 ; 17 \%$ and $55 \%$ respectively for target lesion occlusion, $P<0.001)$. In post-hoc analysis of the data, treatment with a DEB was significantly associated with improved ulcer healing; the index ulcers of $86 \%$ of patients treated with DEB and $67 \%$ of patients treated with PTA had completely healed 12 months after the intervention $(P=0.01)$. These changes in secondary end points led to fewer major adverse events in patients treated with DEB than in PTA-treated patients in the 12 months after angioplasty (31\% and $51 \%$ respectively, $P=0.05$ ).

A longer follow-up period is needed to determine the role of DEB treatment in preventing amputations caused by critical limb ischaemia, as only one amputation event occurred during the 12 month study. Indeed, "vessel patency alone is considered necessary but not sufficient to guarantee amputationfree survival," the authors wrote in their article.

Thomas Zeller, who was not involved in the DEBATE-BTK study, expects that with longer follow-up, reduced amputation rates will be seen in patients treated with DEBs compared with those treated with PTA. In his previously published work on drug-eluting stents (DES) treatment of short stenoses below the knee, Zeller says that he "found only a trend towards improved clinical outcomes for the DES cohort as a result of the better preserved patency; however, after 2 years, the amputation rate and the target lesion revascularization rate were significantly lower in the DES cohort. Similar outcomes can be expected for DEB trials such as DEBATE-BTK." A trial investigating the long-term effects of DES treatment for patients with critical limb ischaemia is already underway.

Megan Cully

Original article Liistro, F. et al. Drug-eluting balloon in peripheral intervention for below the knee angioplasty evaluation (DEBATE-BTK): a randomized trial in diabetic patients with critical limb ischemia. Circulation doi:10.1161/CIRCULATIONAHA.113.001811 